\title{
Desmoplastic fibroma in the proximal femur: A case report with long-term follow-up
}

\author{
YU-BAO GONG ${ }^{1}$, LI-MEI QU ${ }^{2}, \mathrm{XIN}^{1}{ }^{1}$ and JIAN-GUO LIU ${ }^{1}$ \\ Departments of ${ }^{1}$ Bone and Joint Surgery and ${ }^{2}$ Pathology, The First Hospital of Jilin University, \\ Changchun, Jilin 130021, P.R. China
}

Received October 20, 2014; Accepted July 10, 2015

DOI: $10.3892 / \mathrm{ol} .2015 .3603$

\begin{abstract}
Desmoplastic fibroma of the bone is an extremely rare primary benign tumor. The present study reports a case of desmoplastic fibroma of the bone with the longest published follow-up. A 21-year-old female presented to The First Hospital of Jilin University (Changchun, Jilin, China) with thigh pain. Radiography demonstrated a lytic expansile lesion in the proximal femur. Curettage was performed, followed by use of an allogeneic graft. One month later, the patient suffered a pathological fracture and was treated with an open reduction and internal fixation. There was no recurrence of the tumor over a 28-year follow-up period. In conclusion, desmoplastic fibroma in the proximal femur is rare and an intralesional resection is strongly recommended to prevent recurrence. The disease may be misdiagnosed as a bone cyst, so the diagnosis should be confirmed with a histological examination.
\end{abstract}

\section{Introduction}

Desmoplastic fibroma of the bone is an extremely rare, lytic, locally aggressive but non-metastatic, primary bone tumor. Histologically, desmoplastic fibroma of the bone is formed of wavy fibroblasts and abundant collagenous tissue, and bears a close resemblance to a desmoid tumor of the soft tissues. It is a slowly progressing, locally invasive tumor, which exhibits a characteristic production of collagen fibers (1). The most common location is the mandible, followed by the extremities, head and spine. The incidence is estimated at $0.06 \%$ among all bone tumors and $0.3 \%$ among benign bone tumors (1-4). Desmoplastic fibroma of the bone occurs most often in the first three decades of life and is found equally among men and women (5). The most common symptoms include pain and swelling, and $12 \%$ of patients present with pathological fractures. However, a number of patients may be asymptomatic and thus, the tumor

Correspondence to: Dr Jian-Guo Liu, Department of Bone and Joint Surgery, The First Hospital of Jilin University, 71 Xinmin Street, Changchun, Jilin 130021, P.R. China

E-mail: dr.liujianguo@hotmail.com

Key words: desmoplastic fibroma of the bone, fibromatosis, bone is often identified incidentally (6). On radiographs, the lesion is lytic, sometimes poorly limited, and exhibits low signal intensity on T2-weighted magnetic resonance imaging. The diagnosis of the disease is predominantly based on pathological examination. The recurrence rates in patients treated with or without resection are 17 and $55-72 \%$, respectively (6).

The disease was first identified by Jaffe in 1958 (7). To date, $\sim 200$ cases of desmoplastic fibroma of the bone have been reported in the literature. Of these, 13 have been reported in the femur $(8-19)$, with only two in the proximal femur $(8,13)$ and the remaining located in the distal femur $(9-12,14-17,19,20)$. To the best of our knowledge, the current study presents the longest published follow-up of desmoplastic fibroma of the bone. Written informed consent was obtained from the patient.

\section{Case report}

In April 1981, a 21-year-old female presented to The First Hospital of Jilin University (Changchun, Jilin, China) with slight lameness and pain in the proximal right thigh that had persisted for the last month. One day prior to admission, the pain worsened. A physical examination revealed no evident abnormalities, with the exception of tenderness and pain on percussion of the locality. Radiography showed a lytic, cystic, low-density lesion in the proximal femur and femoral neck (Fig. 1). The lesion was expansile and the cortex was slightly eroded. The margins were intact and sclerotic. The initial diagnosis at admission was of a bone cyst, based on radiological evidence, that revealed a well-defined, centrally located, metaphyseal-diaphyseal lucent lesion, which exhibited expansion and thinning of the cortices. The patient underwent curettage and an allogeneic bone graft was applied. Histological examination revealed sparse, spindle-shaped cells with low mitotic activity, surrounded and separated from one another by abundant collagenous tissue. Additionally, there was no necrosis (Fig. 2). Thus, based on the aforementioned postoperative analyses and previous case, a diagnosis of desmoplastic fibroma was determined. The patient was advised to remain on bed-rest for three months. Despite this, one month later, the patient suffered a pathological fracture from a fall while walking (Fig. 3A). Surgery consisting of an open reduction and internal fixation was performed (Fig. 3B). The fracture healed after eight months (Fig. 3C), and the patient's leg was partially weight-bearing. The patient was followed up at one or two year intervals with physical and radiological 


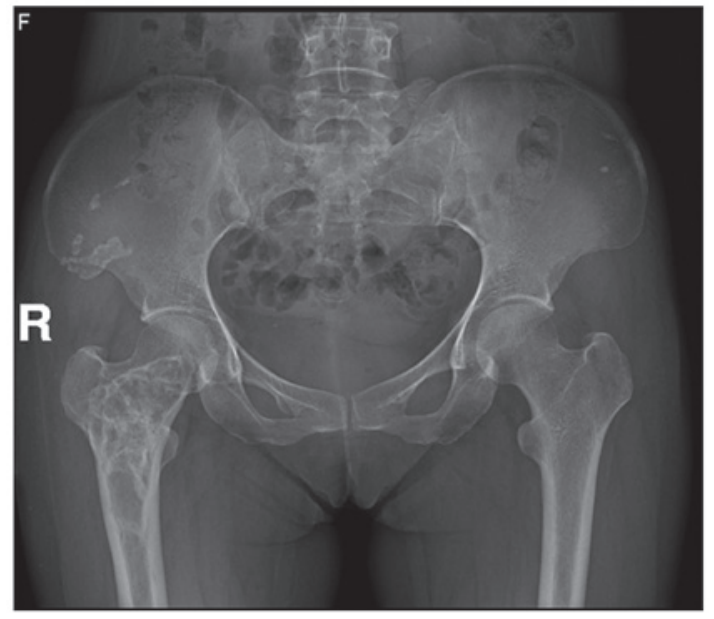

Figure 1. Radiography showing a lytic, cystic, low-density lesion of the proximal femur and femoral neck. The lesion was expansile and the cortex was slight eroded. The lesion exhibited intact, well defined sclerotic margins.

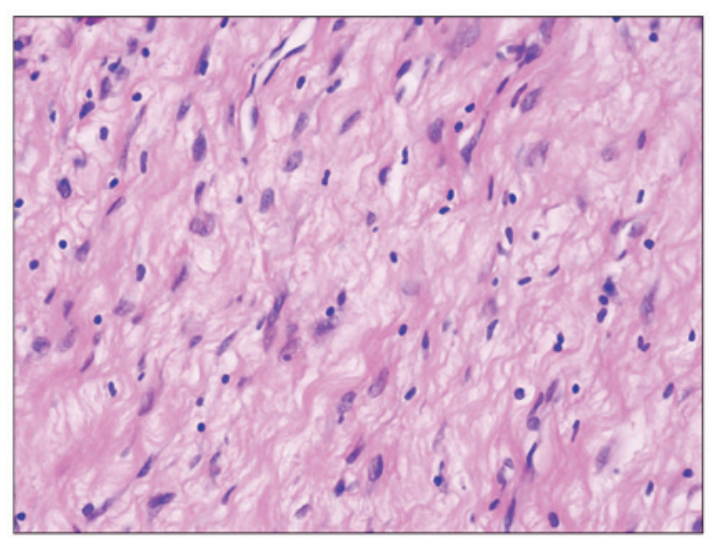

Figure 2. Histological examination revealing sparse spindle-shaped cells with low mitotic activity, surrounded and separated from one another by abundant collagenous tissue, and no necrosis.

examinations. After 28 years of follow-up, the patient was able to bear weight on the leg and has not experienced recurrence.

\section{Discussion}

The present study reports the case of a 21-year-old female with a desmoplastic fibroma who was initially diagnosed with a bone cyst of the proximal femur at the age of 21 , was treated with curettage and an allograft, and did well for 28 years. This case is quite similar to four other published studies of a desmoplastic fibroma of the proximal femur $(8,13)$. In all these reported cases, the patients were all initially diagnosed with bone cysts at young age based on radiological evidence and treated accordingly before the final diagnosis of desmoplastic fibroma was determined. Based on the diagnostic history of previous patients, as well as postoperative analyses, the diagnosis of bone cyst was dismissed in favor of demoplastic fibroma in the present case.

Desmoplastic fibroma of the bone is an extremely rare bone tumor that resembles a desmoid tumor of the soft tissues. In 1958, Jaffe (7) first differentiated this bony lesion from other fibromas of bone, and termed it 'desmoplastic fibroma' based on the presence of fibroblasts and abundant collagenous tissue (21).
A

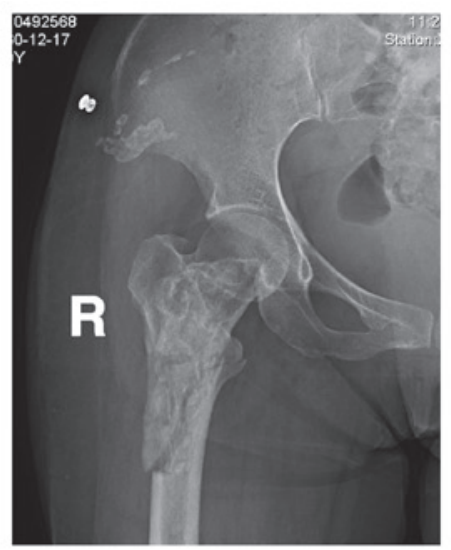

B

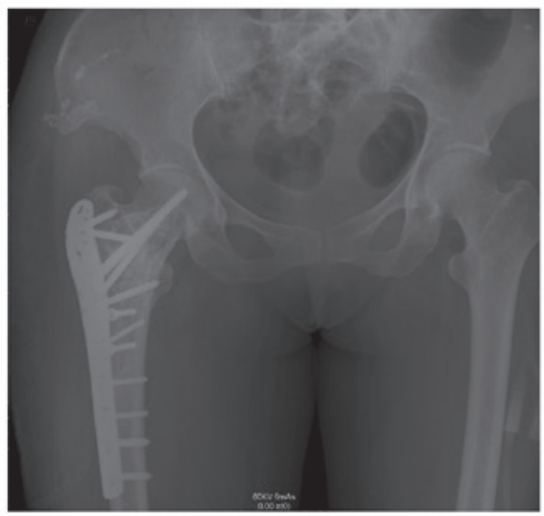

C

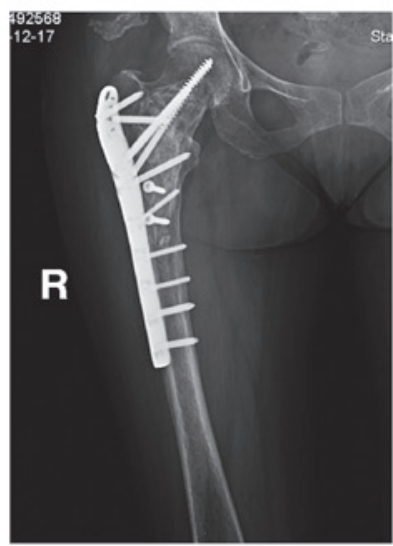

Figure 3. Radiographic findings associated with the fall the patient suffered one month after curettage. (A) Pathological fracture. (B) Following open reduction and internal fixation of the fracture. (C) The healed fracture eight months later.

In comparison to the 13 reported cases of desmoplastic fibroma of the femur (8-20), the patient presented in the current case exhibited desmoplastic fibroma of the proximal femur, which is extremely rare. Among the 13 cases reported previously, only two cases exhibited desmoplastic fibroma of the proximal femur $(8,13)$. Radiographically, the current case presented as a lytic metadiaphyseal lesion, with a honeycomb appearance without matrix mineralization, which is consistent with the radiological manifestations of a bone cyst, as well as the findings of the previous cases. Therefore, diagnosis of desmoplastic fibroma based on radiological evidence alone remains a challenge. The two cases of desmoplastic fibroma of the proximal femur reported previously $(8,13)$ underwent bone curettage and bone grafts, as performed in the present 
case. However, both patients exhibited pathological fractures and subsequently underwent a second surgical procedure for internal fixation $(8,13)$. These findings indicate that for desmoplastic fibroma of the proximal femur, internal fixation must be performed at an early stage, as fibroma may affect the mechanical stability of the bone.

The differential diagnosis of a desmoplastic fibroma should consider aggressive fibromatosis. Aggressive fibromatosis, also known as a desmoid tumor, was described prior to desmoplastic fibroma and is defined as a rare mesenchymal neoplasia composed of collagenous tissue and spindle-shaped cells (7). The majority of desmoid tumors are abdominal (69\%); they can be localized intra-abdominally, within the abdominal wall and also extra-abdominally $(7,21)$. As the intraosseus variant is desmoplastic fibroma, morphological differentiation is unlikely.

The clinical manifestations of desmoplastic fibroma include pain and swelling as the disease progresses. The diagnosis of desmoplastic fibroma by radiography alone is challenging. Plain $\mathrm{X}$-ray shows an osteolytic, expansile, medullary lesion with well-defined sclerotic margins, which is often located in the metaphysis. The cortex is usually thin and there are intralesional trabeculae, resembling soap bubbles. Computed tomography is used to detect intraosseus tumor extension and cortical involvement, while magnetic resonance imaging demonstrates the separation of the intraosseus tumor from the bone, and is used to assess extraosseus tumor growth and for pre-operative planning $(1,2,21)$. The radiologic differential diagnosis includes non-ossifying fibroma, fibrous dysplasia, adamantinoma, bone cysts, giant cell tumors and metastases.

The treatment of desmoplastic fibroma varies, but may include curettage, resection (intralesional, marginal or wide) with or without an allograft, cryosurgery, and amputation in recurrent cases $(1-4,22,23)$. The rates of recurrence are $55-72 \%$ without resection and $17 \%$ with resection $(1,22,24-26)$. Metastases have not been reported. The therapy for desmoplastic fibroma is strongly dependent on the region of the body that is affected and the aggressive nature of the lesion. When a resection is feasible, as in the mandible and long bones, it should be performed, as a resection has the lowest rate of recurrence (1). Nedopil et al (21) suggested that in isolated intraosseus lesions without evidence of extension in adjacent soft tissus, and when resection is associated with a higher risk due to anatomical conditions, a risk-benefit analysis should be performed. In these cases, thorough curettage may be adequate management, as it reduces the surgical duration, has a lower risk of infection and facilitates a faster recovery. Once the decision has been made, close post-operative observation, including clinical and radiographic examination, is necessary to detect a recurrent lesion as early as possible. We believe that curettage and allografts may not be sufficient to treat desmoplastic fibroma and propose an intralesional resection and close follow-up in all cases to prevent recurrence.

In conclusion, desmoplastic fibroma in the femur is rare, particularly in the proximal femur. The disease is easily misdiagnosed and treated as a bone cyst. Management strategies should involve observations of the clinical manifestations and confirmation of the diagnosis with histological examination. To prevent recurrence, an intralesional resection and close follow-up after surgery are strongly recommended. In the present case, there was no recurrence of the tumor over a 28-year follow-up period.

\section{References}

1. Böhm P, Kröber S, Greschniok A, Laniado M and Kaiserling E: Desmoplastic fibroma of the bone. A report of two patients, review of the literature and therapeutic implications. Cancer 78: 1011-1023, 1996.

2. Rabin D, Ang LC, Megyesi J, Lee DH and Duggal N: Desmoplastic fibroma of the cranium: Case report and review of the literature. Neurosurgery 52: 950-954, 2003.

3. Ikeshima A and Utsunomiya T: Case report of intra-osseous fibroma: A study on odontogenic and desmoplastic fibromas with a review of the literature. J Oral Sci 47: 149-157, 2005

4. Said-Al-Naief N, Fernandes R, Louis P, Bell W and Siegal GP: Desmoplastic fibroma of the jaw: A case report and review of literature. Oral Surg Oral Med Oral Pathol Oral Radiol Endod 101: 82-94, 2006.

5. Crim JR, Gold RH, Mirra JM, Eckardt JJ and Bassett LW: Desmoplastic fibroma of bone: Radiographic analysis. Radiology 172: 827-832, 1989.

6. Hauben E and Cleton-Jansen AM: Fibrogenic tumours: desmoplastic fibroma of bone. In: WHO Classification of Tumours of Soft Tissue and Bone. Fletcher CDM, Bridge JA, Hogendoorn P and Mertens F (eds). Vol 5. 4th edition. IARC, Lyon, p298, 2013.

7. Jaffe HL: Tumors and Tumorous Conditions of the Bones and Joints. Lea and Febiger, Philadelphia, 1958.

8. Lichtman EA and Klein MJ: Case report 302. Desmoplastic fibroma of the proximal end of the left femur. Skeletal Radiol 13: 160-163, 1985.

9. Wadhwa V, Suh KJ, Yi JH and Chhabra A: Incidental lesion in the femoral metaphysis. Desmoplastic fibroma of the bone. Skeletal Radiol 42: 1739-1740, 1775-1776, 2013.

10. Clayer M and Oakeshott R: Clin Orthop Relat Res. Allograft bone in the treatment of desmoplastic fibroma. A case report. Clin Orthop Relat Res: 219-224, 1994.

11. Rastogi S, Varshney MK, Trikha V, Khan SA and Mittal R: Desmoplastic fibroma: A report of three cases at unusual locations. Joint Bone Spine 75: 222-225, 2008.

12. Chan KW, Pun WK and Choi CH: Desmoplastic fibroma of bone. Pathology 19: 201-203, 1987.

13. Grube-Pagola P and Valle-Landa JC: Desmoplastic fibroma of the femur. Radiologia 55: 359-361, 2013 (In Spanish).

14. Doya H and Yaoita M: Case of desmoplastic fibroma in the femur. Seikei Geka 17: 754-756, 1966 (In Japanese).

15. Matsumori S, Watabe E, ToyoshimaY and Yagi Y: Primary desmoplastic fibroma of the femur. Seikei Geka 23: 275-281, 1972.

16. Bertoni F, Calderoni $\mathrm{P}, \mathrm{Bacchini} \mathrm{P}$ and Campanacci $\mathrm{M}$ : Desmoplastic fibroma of bone. A report of six cases. J Bone Joint Surg Br 66: 265-268, 1984.

17. Petrovichev NN, Filippova NA, Annamukhammedov A and Karapetian RM: Case of desmoplastic fibroma of the femur. Arkh Patol 47: 55-58, 1985 (In Russian).

18. Nielsen GP, O'Connell JX, Dickersin GR and Rosenberg AE: Collagenous fibroma (desmoplastic fibroblastoma): A report of seven cases. Mod Pathol 9: 781-785, 1996.

19. Gao S, Cai Q, Yao W, Wang J, Zhang P and Wang X: Desmoplastic (collagenous) fibroma of the femur: A case report and review of the literature. Oncol Lett 6: 1285-1288, 2013

20. Yokouchi M, Ueno Y, Nagano S, et al: Extended curettage and heat ablation for desmoplastic fibroma of the distal femur with a 12-year follow-up period: A case report. Oncol Lett 8: 11031106, 2014

21. Nedopil A, Raab P and Rudert M: Desmoplastic fibroma: A case report with three years of clinical and radiographic observation and review of the literature. Open Orthop J 8: 40-46, 2013.

22. Papagelopoulos PJ, Mavrogenis AF, Mitsiokapa EA, et al: Current trends in the management of extra-abdominal desmoid tumours. World J Surg Oncol 4: 21, 2006.

23. Daneyemez M, Akay KM and Izci Y: Desmoplastic fibroma of the cervical spine. Eur Spine J 14: 799-802, 2005.

24. Gedikoglu G, Aksoy MC and Ruacan S: Fibrocartilaginous mesenchymoma of the distal femur: Case report and literature review. Pathol Int 51: 638-642, 2001.

25. Iatrou IA, Theologie-Lygidakis N and Leventis MD: Case report: Desmoplastic fibroma of the mandible in a child presenting with TMJ dysfunction. Eur Arch Paediatr Dent 9: 105-108, 2008.

26. Perlick L, Zander D, Wallny T and Zhou H: Desmoplastic fibroma of the fibula. A difficult clinical, radiological and histological diagnosis. Zentralbl Chir 125: 895-899, 2000 (In German). 